Arq. Bras. Med. Vet. Zootec., v.68, n.1, p.208-214, 2016

\title{
Termorregulação de pintos de um dia submetidos a ambiente térmico simulado de transporte
}

\author{
[Thermoregulation of day-old chickens submitted to simulated \\ transport thermal environment] \\ F.M.C. Vieira ${ }^{1}$, I.J.O. Silva ${ }^{2}$, A.C. Nazareno ${ }^{2}$, P.N. Faria ${ }^{3}$, K.O.S. Miranda ${ }^{4}$ \\ ${ }^{1}$ Grupo de Estudos em Biometeorologia - GEBIOMET - Universidade Tecnológica Federal \\ do Paraná - Dois Vizinhos, PR \\ ${ }^{2}$ Núcleo de Pesquisa em Ambiência - NUPEA - USP/ESALQ - Piracicaba, SP \\ ${ }^{3}$ Faculdade de Matemática - Universidade Federal de Uberlândia - Uberlândia, MG \\ ${ }^{4}$ USP/ESALQ - Piracicaba, SP
}

\begin{abstract}
RESUMO
Objetivou-se, por meio deste trabalho, avaliar a termorregulação de pintos de um dia, mantidos em caixas de transporte, sob condição térmica simulada de transporte. Dois experimentos replicados executados em dias diferentes foram conduzidos em câmara climática. Para cada um dos experimentos, foram utilizados 360 pintos de um dia. Esses animais foram submetidos às condições de frio, conforto e calor, durante uma hora em cada tratamento. Os animais foram mantidos em caixas de transporte de pintos de um dia com quatro compartimentos, sendo cada um deles com 10 animais, totalizando 40 animais por caixa. Foram utilizadas três caixas empilhadas, simulando o que ocorre no caminhão de transporte. Para avaliação fisiológica, foram realizadas as seguintes medidas: frequência respiratória, peso, temperaturas superficial e cloacal. No geral, o posicionamento das caixas não alterou profundamente o conforto térmico dos pintos de um dia. Com relação às faixas térmicas, a condição de frio foi a que mais afetou as respostas fisiológicas, com destaque para as temperaturas superficial média e cloacal. No entanto, com o aumento do tempo de exposição, possivelmente o tratamento térmico por calor possa ter mais efeito sobre a termorregulação desses animais.
\end{abstract}

Palavras-chave: frangos de corte, logística, bem-estar animal, pintos de um dia

\begin{abstract}
An experiment was conducted to assess the thermoregulation of day-old chickens, placed in transport boxes, under simulated transport thermal condition. Two replicated trials were done in two different days in a windowless environmentally controlled chamber. For each one, a total of 360 one-day-old broiler chicks were used. These animals were submitted to three thermal conditions (cold, thermoneutral and heat), for $1 \mathrm{~h}$ each. The birds were placed in boxes for day-old chick transport, divided in 40 chicks per box, with three boxes stacked vertically, for each treatment (120 chicks per treatment). Body weight, respiratory frequency, mean surface and cloacal temperature were monitored in a sample of 20 animals per box, with measurements were made before and after the treatments. The box placement had a negligible effect on the thermal comfort of birds. The mean surface and cloacal temperature responses were markedly affected by the cold treatment. However, with an increasing exposure time, the heat treatment might possibly have a clear evidence on the thermoregulation of day-old chickens.
\end{abstract}

Keywords: broilers, logistics, animal welfare, neonatal chicks

Recebido em 29 de julho de 2014

Aceito em 16 de setembro de 2015

E-mail: fredericovieira@utfpr.edu.br 


\section{INTRODUÇÃO}

O desempenho de frangos de corte ao longo da cadeia produtiva está ligado ao manejo dispensado aos pintos de um dia, desde o nascimento até o alojamento nas granjas. No entanto, muitos pontos críticos relacionados ao conforto térmico e bem-estar animal são observados nessas etapas, especialmente durante o transporte do incubatório à granja.

O ambiente térmico da sala após a eclosão modula todo o mecanismo fisiológico de controle térmico para todas as fases posteriores de desenvolvimento (Tzschentke, 2007). A temperatura ideal dos pintos na primeira semana de idade encontra-se entre 32 e $35^{\circ} \mathrm{C}$, e a umidade em torno de $60 \%$ (Lin et al., 2005; Shinder et al., 2007). Temperaturas abaixo ou acima desse intervalo afetam a saúde e o desempenho dos animais.

Em condições de frio foram observados quadros de ascite e redução de peso corporal aos sete dias (Malheiros et al., 2000; Mujahid e Furuse, 2009). Sob elevadas temperaturas pode ocorrer desidratação, aumento da temperatura cloacal e frequência respiratória, bem como variações no peso corporal na primeira semana (Dionello et al., 2002). Lin et al. (2005) observaram que a diferença entre a temperatura superficial de pintos na primeira semana e a temperatura ambiente elevada foi menor do que em animais mantidos em condição de conforto. Os mesmos autores sugeriram que a capacidade de perda de calor sensível pelas aves jovens também é reduzida com o aumento da temperatura, consequentemente resultando em aumento da temperatura cloacal e utilização de mecanismos de perda de calor latente.

No entanto, observa-se uma quantidade limitada de informações sobre as trocas térmicas de pintos de um dia mantidos em caixas de transporte e os efeitos térmicos do ambiente de transporte no conforto desses animais. Dessa forma, objetivouse, por meio deste trabalho, avaliar a termorregulação de pintos de um dia, mantidos em caixas de transporte, sob condição térmica simulada de transporte.

\section{MATERIAL E MÉTODOS}

Dois experimentos replicados executados em dias diferentes foram conduzidos em câmara climática. Devido ao fato de as caixas não terem sido submetidas à simulação de movimento, denominou-se apenas a simulação como sendo condição térmica simulada. Para cada um dos experimentos, foram utilizados 360 pintos de um dia da linhagem Cobb 500, devidamente sexados e vacinados, provenientes de matrizes com idade de 41 semanas. Os ovos incubados foram do mesmo lote de matrizes, mantidos na mesma incubadora e nascedouro no incubatório. Esses animais foram submetidos a três condições térmicas durante uma hora, de acordo com as faixas propostas por Malheiros et al. (2000), Lin et al. (2005) e Shinder et al. (2007) (Tab. 1).

Tabela 1. Faixas térmicas de conforto e estresse para pintos de um dia

\begin{tabular}{ccc} 
Faixas térmicas & $\begin{array}{c}\text { Temperatura } \\
\left({ }^{\circ} \mathrm{C}\right)\end{array}$ & $\begin{array}{c}\text { Umidade } \\
\text { relativa }(\%)\end{array}$ \\
\hline Conforto térmico & 35 & 60 \\
Estresse por frio & 15 & 60 \\
Estresse por calor & 40 & 70 \\
\hline
\end{tabular}

A justificativa em se trabalhar por uma hora refere-se ao fato de o tempo não ter sido fator de estudo nessa etapa. Dessa forma, a escolha de um tempo fixo possibilitou apenas a observações das faixas térmicas e do posicionamento das caixas.

Os animais foram mantidos em caixas de transporte de pintos de um dia com quatro compartimentos, sendo cada um deles com 10 animais, totalizando 40 animais por caixa (Fig. 1a). Foram utilizadas três caixas empilhadas (superior, mediana e inferior), simulando o que ocorre no caminhão de transporte (Fig. 1b).

Esse tipo de caixa foi utilizado devido à disponibilidade dela no local da pesquisa, além da possibilidade de dividir o lote em machos e fêmeas. Posteriormente, decidiu-se trabalhar com lote misto, mantendo os animais nesse tipo de caixa. 


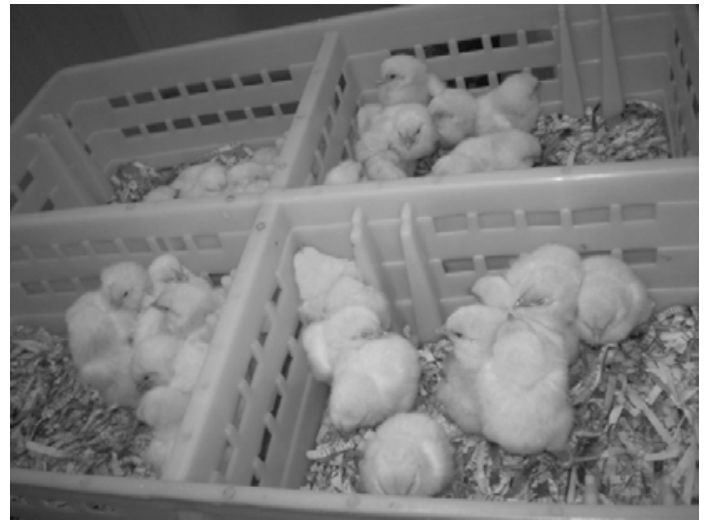

(a)

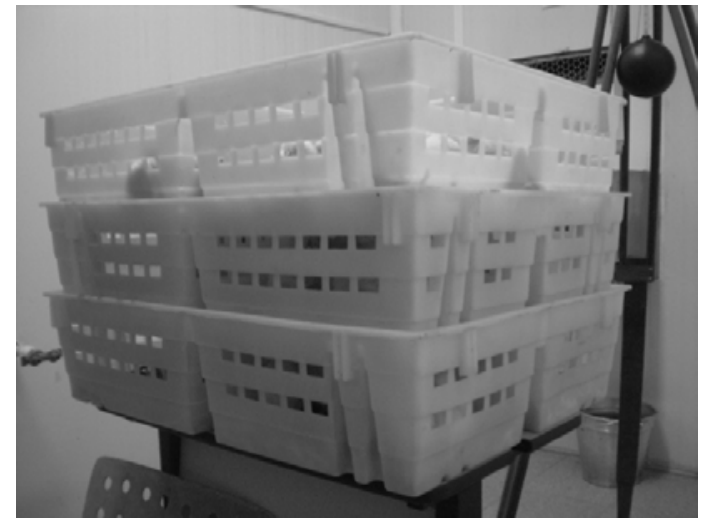

(b)

Figura 1. Distribuição de pintos de um dia nas caixas de transporte (a) e caixas empilhadas dentro da câmara climática (b).

Antes dos tratamentos, as caixas foram mantidas em um círculo de proteção com aquecimento, para o retorno da condição de conforto dos animais após o transporte do incubatório para o laboratório. Após os tratamentos na câmara climática, os animais foram mantidos em um círculo de proteção, com aquecimento por meio de campânula com lâmpadas e alimentação e fornecimento de água ad libitum. O intuito de mantê-los no laboratório foi verificar se houve mortalidade no dia seguinte do experimento, conforme explicado adiante.

Para avaliação fisiológica, as medidas foram aferidas logo após uma hora de tratamento térmico, e as medições das variáveis obedeceram a um ordenamento para não influenciar nas respostas fisiológicas, conforme a ordem adotada a seguir: frequência respiratória, peso, temperatura superficial e temperatura cloacal. A frequência respiratória (FR, mov/min) se deu a partir da contagem do número de movimentos abdominais realizados pelo pinto pelo tempo de 15 segundos, que posteriormente foram multiplicados por quatro para se ter a medida em movimentos por minuto. Foram realizados monitoramentos do peso corporal (PC, g) utilizando-se uma balança semianalítica, com precisão de $0,1 \mathrm{~g}$. A temperatura da superfície $\left({ }^{\circ} \mathrm{C}\right)$ se deu pela aferição da temperatura da asa, cabeça, pata e dorso do pinto de um dia utilizando-se um termômetro de infravermelho. Com base nesses pontos, foi possível calcular a temperatura média superficial $\left(\mathrm{TSM},{ }^{\circ} \mathrm{C}\right)$, por meio da equação proposta por Nascimento
(2010), para frangos de corte da linhagem Cobb na primeira semana de criação:

$$
T S M=3,47+0,11 T_{w}+0,10 T_{h}+0,15 T_{l}+0,56 T_{b}
$$

em que: $\mathrm{Tw}=$ temperatura da asa; $\mathrm{Th}=$ temperatura da cabeça; $\mathrm{Tl}=$ temperatura da perna e $\mathrm{Tb}=$ temperatura do dorso.

A temperatura cloacal $\left(\mathrm{TC},{ }^{\circ} \mathrm{C}\right)$ foi aferida com um termômetro do tipo $\mathrm{T}$ introduzido na cloaca do pinto até a sua estabilização e obtenção do valor da temperatura.

A mortalidade (\%) foi calculada pelo número de aves mortas em relação ao total de aves transportadas durante o experimento e um dia após o ensaio, para verificar o efeito a posteriori da combinação dos tratamentos. Esse dado foi obtido separadamente para cada tratamento.

Foi realizada uma análise descritiva utilizando-se médias e erros-padrão da média para os fatores avaliados, por meio do software estatístico Minitab v.15 (MINITAB 15 STATISTICAL SOFTWARE, 2007). A estrutura de parcelas adotada neste trabalho foi o delineamento em blocos ao acaso, sendo cada experimento replicado considerado um bloco, ou seja, cada dia em que foi realizado um experimento replicado foi considerado bloco. A estrutura de tratamento adotada foi um fatorial $3 \times 3$ (três faixas térmicas e três posições de caixas). Para a análise estatística confirmatória, foi realizada uma análise de variância seguida do teste de Tukey a $5 \%$ de probabilidade para a comparação 
múltipla de médias, visando encontrar possíveis diferenças entre os tratamentos, no que diz respeito ao peso corporal, à frequência respiratória, à temperatura média superficial e à temperatura cloacal. Tais análises foram realizadas por meio do software estatístico SAS 9.2 (SAS INSTITUTE, 2009).

\section{RESULTADOS E DISCUSSÃO}

Em geral, houve pouca variação dos fatores estudados com relação ao tratamento caixa, o que evidencia um possível efeito não significativo de caixas dentro de cada tratamento para a maioria das variáveis estudadas (Tab. 2).

Tabela 2. Média e erro-padrão da média para frequência respiratória (RF), peso corporal (PC), temperatura superficial média (TSM) e temperatura cloacal (TC) em relação aos tratamentos térmicos e à posição das caixas

\begin{tabular}{cccccc}
\hline \multirow{2}{*}{ Faixas } & \multirow{2}{*}{ Caixas } & \multicolumn{4}{c}{ Variáveis } \\
\\
& 1 & FR $(\mathrm{mov} / \mathrm{min})$ & $\mathrm{PC}(\mathrm{g})$ & $\mathrm{TSM}\left({ }^{\circ} \mathrm{C}\right)$ & $\mathrm{TC}\left({ }^{\circ} \mathrm{C}\right)$ \\
\hline \multirow{3}{*}{ Conforto térmico } & 2 & $68 \pm 0,26$ & $40,9 \pm 0,08$ & $35,1 \pm 0,01$ & $41,2 \pm 0,01$ \\
& 3 & $65 \pm 0,20$ & $41,4 \pm 0,10$ & $35,2 \pm 0,01$ & $41,2 \pm 0,01$ \\
\multirow{3}{*}{ Estresse por frio } & 1 & $124 \pm 0,43$ & $41,1 \pm 0,10$ & $35,0 \pm 0,01$ & $40,9 \pm 0,01$ \\
& 2 & $124 \pm 0,23$ & $41,0 \pm 0,10$ & $21,2 \pm 0,04$ & $37,8 \pm 0,03$ \\
\multirow{2}{*}{ Estresse por calor } & 3 & $130 \pm 0,28$ & $40,4 \pm 0,11$ & $21,4 \pm 0,03$ & $37,6 \pm 0,02$ \\
& 1 & $225 \pm 1,20$ & $39,7 \pm 0,07$ & $38,0 \pm 0,01$ & $42,4 \pm 0,01$ \\
& 2 & $242 \pm 0,59$ & $39,7 \pm 0,09$ & $38,2 \pm 0,01$ & $42,6 \pm 0,01$ \\
& 3 & $252 \pm 0,49$ & $40,9 \pm 0,09$ & $38,1 \pm 0,01$ & $42,4 \pm 0,01$ \\
\hline
\end{tabular}

A variação mais acentuada foi detectada entre caixas quanto à frequência respiratória no tratamento de calor, indicando que a pior condição foi encontrada na caixa 3 . O fator peso manteve-se constante em todos os tratamentos (térmicos e entre caixas), sem variação ao longo da pesquisa. Com relação à mortalidade, não foi observada neste presente estudo nenhum óbito dos animais durante os tratamentos térmicos e nas diferentes caixas. Segundo Shinder et al. (2007), as aves jovens não podem manter a temperatura corporal elevada em situação de frio por mais de 90 minutos sem que haja aumento na mortalidade do lote. O tempo de duração dos tratamentos foi de uma hora cada um, o que justificou a sobrevivência dos animais durante a pesquisa.

Houve interação entre os tratamentos térmicos e caixas, o que evidencia a influência das condições térmicas na alteração da frequência respiratória dos animais. Entretanto, o mesmo fato não ocorreu em relação às posições de caixas (Tab. 3).

Tabela 3. Valores médios da interação entre posição das caixas e faixas térmicas em relação à frequência respiratória de pintos de um dia

\begin{tabular}{cccc}
\hline \multirow{2}{*}{ Caixas } & \multicolumn{3}{c}{ Faixas térmicas } \\
\cline { 2 - 4 } & Conforto térmico & Estresse por frio & Estresse por calor \\
\hline 1 & $68 \mathrm{bA}$ & $124 \mathrm{aA}$ & $225 \mathrm{aB}$ \\
2 & $64 \mathrm{bA}$ & $124 \mathrm{aA}$ & $242 \mathrm{aA}$ \\
3 & $65 \mathrm{cA}$ & $130 \mathrm{bA}$ & $252 \mathrm{aA}$ \\
\hline
\end{tabular}

Médias com letras diferentes (maiúsculas nas colunas e minúsculas nas linhas) diferem entre si pelo teste Tukey $(\mathrm{P}<0,05)$.

Com relação às caixas dentro de cada faixa térmica, foi possível constatar que não houve diferença entre caixas para os tratamentos conforto e frio. No tratamento calor, nota-se a diferença entre as caixas 1 e 2 , bem como entre a 1 e 3 . Ao se compararem os tratamentos térmicos dentro de cada caixa, verificou-se que houve diferença entre todas as combinações de tratamentos térmicos na caixa 3 , evidenciando também que nessa posição a condição mais crítica para os pintos foi percebida no tratamento de estresse térmico por calor. 
Numa situação de estresse agudo por calor, um dos meios mais eficientes visando à redução da temperatura interna é o ofego, processo que representa $75 \%$ da perda de calor latente das aves (Silva, 2008). Em um ambiente restrito para as trocas térmicas, como, por exemplo, uma caixa de transporte, o nível de umidade relativa aumenta nesses casos, o que dificulta o processo de troca térmica (Kettlewell et al., 2000; Oliveira et al., 2006). Isto provoca aumento da frequência respiratória, seguido de estresse, que tende a aumentar se a temperatura se mantiver elevada por mais tempo. No entanto, a caixa 1 possui as laterais e a parte superior para ventilação ou troca de ar, ao contrário das caixas 2 e 3 , que só possuem as laterais. Desse modo justificaram-se os valores maiores de frequência respiratória nas diferentes faixas térmicas nas caixas inferiores, bem como a maior variação entre os valores de frequência respiratória nos diversos tratamentos térmicos na caixa 3. No transporte comercial de pintos de um dia, em um baú climatizado, são três pilhas sobrepostas de caixas de transporte de cada lado, sendo cada pilha composta de cinco a seis caixas e com 10 a 15 fileiras longitudinais. Assim, as caixas inferiores podem proporcionar maior desconforto às aves em ambientes com elevadas temperaturas.

Com relação à variável peso, não houve efeito de nenhum dos fatores avaliados neste estudo, fato esse justificado pela baixa variabilidade entre os animais, conforme mostrado na Tab. 2.

Quanto à temperatura superficial, nota-se que não houve interação entre as faixas térmicas e a posição das caixas e o efeito de caixas. No entanto, observou-se efeito de faixas térmicas na variação da temperatura superficial média (Tab. 4).
Tabela 4. Temperatura superficial média para pintos de corte submetidos às diferentes faixas térmicas

\begin{tabular}{cc}
\hline \multirow{2}{*}{ Faixas térmicas } & $\begin{array}{c}\text { Temperatura superficial } \\
\text { média }\end{array}$ \\
\cline { 2 - 2 } & $\left({ }^{\circ} \mathrm{C}\right)$ \\
\hline Estresse por calor & $38,1 \mathrm{a}$ \\
Conforto térmico & $35,1 \mathrm{~b}$ \\
Estresse por frio & $21,3 \mathrm{c}$ \\
\hline
\end{tabular}

Médias com letras minúsculas diferentes nas colunas diferem entre si pelo teste Tukey $(\mathrm{P}<0,05)$.

A maior perda de calor sensível dos pintos de um dia foi observada no tratamento de estresse por frio, cujo gradiente de temperatura da superfície comparado com a situação de conforto alcançou $13,8^{\circ} \mathrm{C}$. A diferença entre o tratamento térmico de conforto e o estresse por calor foi de $3^{\circ} \mathrm{C}$. Resultados similares foram encontrados por Lin et al. (2005), os quais discutiram que a temperatura superficial de pintos submetidos aos tratamentos com elevada temperatura é similar à temperatura ambiente, o que explica a habilidade reduzida em perder energia térmica na forma sensível sob temperaturas elevadas. No presente estudo, a temperatura superficial média no tratamento de estresse por calor esteve próxima da temperatura ambiente (aproximadamente $40^{\circ} \mathrm{C}$ ), o que confirma os estudos anteriores. Por outro lado, o gradiente térmico entre a superfície e $\mathrm{o}$ ar em condição de frio se ampliou consideravelmente nesta pesquisa, devido à baixa capacidade termorregulatória de pintos de um dia e à facilidade de perder energia térmica para um ambiente mais frio (Gustin, 2003).

No referente à temperatura cloacal, foi observada interação entre as faixas térmicas e a posição das caixas. Porém, não houve diferença entre caixas para nenhum dos tratamentos térmicos (Tab. 5).

Tabela 5. Valores médios da interação entre posição das caixas e faixas térmicas em relação à temperatura cloacal de pintos de um dia

\begin{tabular}{cccc}
\hline \multirow{2}{*}{ Caixas } & \multicolumn{3}{c}{ Faixas térmicas } \\
\cline { 2 - 4 } & Conforto térmico & Estresse por frio & Estresse por calor \\
\hline 1 & $41,2 \mathrm{bA}$ & $37,8 \mathrm{cA}$ & $42,4 \mathrm{aA}$ \\
2 & $41,2 \mathrm{bA}$ & $37,6 \mathrm{cA}$ & $42,6 \mathrm{aA}$ \\
3 & $40,9 \mathrm{bA}$ & $37,7 \mathrm{cA}$ & $42,4 \mathrm{aA}$ \\
\hline
\end{tabular}

Médias com letras diferentes (maiúsculas nas colunas e minúsculas nas linhas) diferem entre si pelo teste Tukey $(\mathrm{P}<0,05)$.

Os tratamentos térmicos diferiram entre si dentro de cada caixa, sendo o efeito mais notável no tratamento de estresse por frio, cujo gradiente de temperatura entre a situação de conforto e de frio foi de aproximadamente $3,2^{\circ} \mathrm{C}$. Ao se comparar o tratamento de estresse por calor com o de 
conforto, a diferença foi de apenas $1,5^{\circ} \mathrm{C}$. Segundo resultados anteriores para animais com três dias de idade, temperaturas do ar reduzidas como as do presente estudo forçam bastante a demanda de manutenção de temperatura dos pintos de um dia (Shinder et al., 2007). Devido à falta de empenamento conjugada com mecanismos termorregulatórios incompletos, a temperatura cloacal tende a se reduzir bruscamente ao longo do tempo de exposição a ambientes frios (Dunnington e Siegel, 1984; Lin et al., 2005).

Em geral, com base nos resultados deste presente trabalho, é possível afirmar que o posicionamento das caixas não alterou profundamente o conforto térmico dos pintos de um dia. Possivelmente, o tamanho da pilha da caixa foi insuficiente para se observar o efeito na termorregulação desses animais. No entanto, a ocorrência da diferença entre as caixas 2 e 3 na frequência respiratória durante o tratamento de estresse por calor é um indicativo de um possível efeito das caixas, pois, devido aos diferentes microambientes com ventilação diferenciada, esses efeitos também podem ser diferenciados.

Com relação às faixas térmicas, nota-se o efeito significativo da temperatura conjugada com a umidade relativa no conforto térmico de pintos de um dia em situação de transporte. A condição de frio foi a que mais afetou as respostas fisiológicas, com destaque nas temperaturas superficial média e cloacal. No entanto, com o aumento do tempo de exposição, possivelmente o tratamento térmico por calor possa ter mais efeito sobre a termorregulação desses animais, indicado no presente estudo apenas por meio da frequência respiratória. Apesar de a preocupação com pintos de um dia ser maior em relação ao estresse por frio, o estresse por calor pode causar danos na capacidade termorregulatória dos animais (Dionello et al., 2002). Na situação de transporte, poucas informações subsidiam o setor produtivo para a tomada de decisão quanto às condições ideais de temperatura e umidade relativa no interior dos caminhões climatizados. Mesmo assim, deve-se preocupar com o controle das temperaturas ideais. Em países de clima tropical, o controle do ambiente interno dos caminhões transportadores geralmente excede as temperaturas ótimas recomendadas, seja pela falta de um isolamento térmico na estrutura dos caminhões seja pelo controle ineficiente dos sistemas de climatização.

\section{CONCLUSÃO}

No que diz respeito ao posicionamento das caixas de transporte, não foram verificadas variações desse fator nas respostas físiológicas e produtivas dos animais. Com relação às condições térmicas, a maior influência verificada foi observada no estresse pelo frio, por meio da temperatura superficial média e da temperatura cloacal, seguida pelo estresse por calor, com relação à frequência respiratória.

\section{AGRADECIMENTOS}

Os autores agradecem à Fundação de Amparo à Pesquisa do Estado de São Paulo (Fapesp), pelo apoio financeiro a esta pesquisa e à Capes, pela concessão da bolsa de estudo; à médica veterinária Lígia Laís Rodrigues da Silva, à zootecnista Ana Paula Dammski e ao engenheiro agrônomo Thiago Ansante, pela colaboração no registro dos dados; ao Prof. Dr. Lúcio Borges de Araújo, pelo auxílio nas análises estatísticas.

\section{REFERÊNCIAS}

DIONELLO, N.J.L.; MACARI, M.; FERRO, J.A. et al. Respostas fisiológicas associadas à termotolerância em pintos de corte de duas linhagens por exposição a altas temperaturas. Rev. Bras. Zootec., v.31, p.79-85, 2002.

DUNNINGTON, E.A.; SIEGEL, P.B. Thermoregulation in newly hatched chicks. Poultry Sci., v.63, p.1303-1313, 1984.

GUSTIN, P.C. Manejo dos pintos no incubatório, expedição, transporte e alojamento na granja. In: MACARI, M.; GONZALES, E. (Eds.). Manejo da incubação. Jaboticabal: FACTA, 2003. 537p.

KETTLEWELL, P.J.; HOXEY, R.P.; MITCHELL, M.A. Heat produced by broiler chickens in a commercial transport vehicle. $J$. Agric. Eng. Res., v.75, p.315-326, 2000.

LIN, H.; ZHANG, H.F.; JIAO, H.C. et al. Thermoregulation responses of broiler chickens to humidity at different ambient temperatures. I. one week of age. Poultry Sci., v.84, p.1166-1172, 2005. 
MALHEIROS, R.D.; MORAES, V.M.B.; BRUNO, L.D.G. et al. Environmental temperature and cloacal and surface temperatures of broiler chicks in first week post-hatch. J. Appl. Poultry Res., v.9, p.111-117, 2000.

MINITAB 15 statistical software. Computer software. Stage College: Minitab, Inc., 2007.

MUJAHID, A.; FURUSE, M. Oxidative damage in different tissues of neonatal chicks exposed to low environmental temperature. Comp. Bioch. Physiol. A, v.152, p.604-608, 2009.

NASCIMENTO, S.T. Determinação do balanço de calor em frangos de corte por meio das temperaturas corporais. 2010. 120f. Dissertação (Mestrado em Física do Ambiente Agrícola) Escola Superior de Agricultura "Luiz de Queiroz", Universidade de São Paulo, Piracicaba, SP.
OLIVEIRA, R.F.M.; DONZELE, J.L.; ABREU, M.L.T. et al. Efeitos da temperatura e da umidade relativa sobre $\mathrm{o}$ desempenho $\mathrm{e} o$ rendimento de cortes nobres de frangos de corte de 1 a 49 dias de idade. Rev. Bras. Zootec., v.35, p.797-803, 2006.

STATISTICAL Analysis System - SAS. Version9.2. Cary: SAS Institute Inc., 2009.

SHINDER, D.; RUSAL, M.; TANNY, J. et al. Thermoregulatory responses of chicks (Gallus domesticus) to low ambient temperatures at an early age. Poultry Sci., v.86, p. 2200-2209, 2007.

SILVA, R.G. Biofísica ambiental: os animais e seu ambiente. Jaboticabal: FUNEP, 2008, 393p.

TZSCHENTKE, B. Attainment of thermoregulation as affected by environmental factors. Poultry Sci., v.86, p.1025-1036, 2007. 